\title{
Improvement of Time Mode Mössbaver Spectrometer by Employing a Crystal Oscillator and Preset Counters
}

\author{
Hideki MiYaJIMA and Shuichi IIDA \\ Department of Physics, Faculty of Science, University of Tokyo \\ Bunkyoku, Tokyo, Japan \\ Received April 26, 1971
}

In a time mode Mössbauer spectrometer, there are two important factors for the improvement of signal to noise ratio, one is the synchronization between the stroage channels and the velocity, and the other is the stability of the spectrometer. Stable triangle and square wave generators have been described by Krankeleit ${ }^{11}$ and by Cohen ${ }^{21}$. In the Mössbauer spectrometer of Elron Co. Ltd., the velocity signal is made from crystalcontrolled clock by a D-A converter. However, these methods are rather complicated.

In this note the authors report a time mode Mössbauer spectrometer, in which all signals for driving the velocity transducer and the multichannel analyser (MCA) are made from a stable crystal clock by employing digital circuits, instead of using wave generators or D-A converters.

A block diagram of apparatus is given in Fig. 1. The MCA drive unit generates an exact sine wave to drive the velocity vibrator. It also generates trigger pulses (start pulse, dwell advance pulse and stop pulse) to oper-

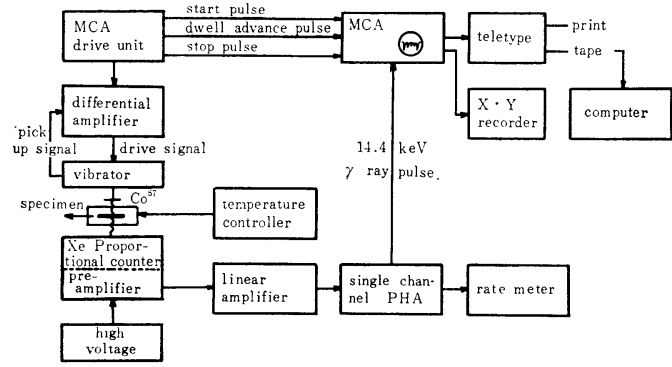

Fig. 1 A block diagram of the Mössbauer spectrometer. ate the MCA. Other equipments are almost same as those of the usual Mössbauer spectrometer.

A block diagram of the MCA drive unit and a schematic time chart are shown in

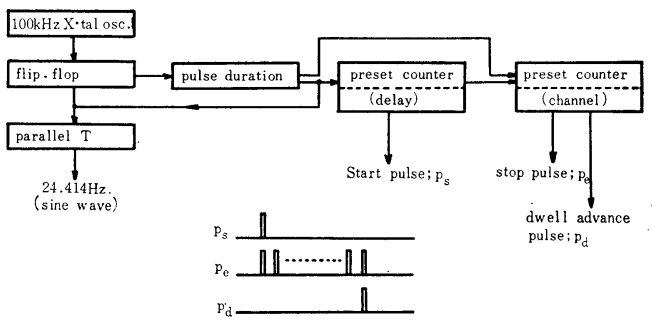

Fig. 2 A block diagram of the MCA drive unit.
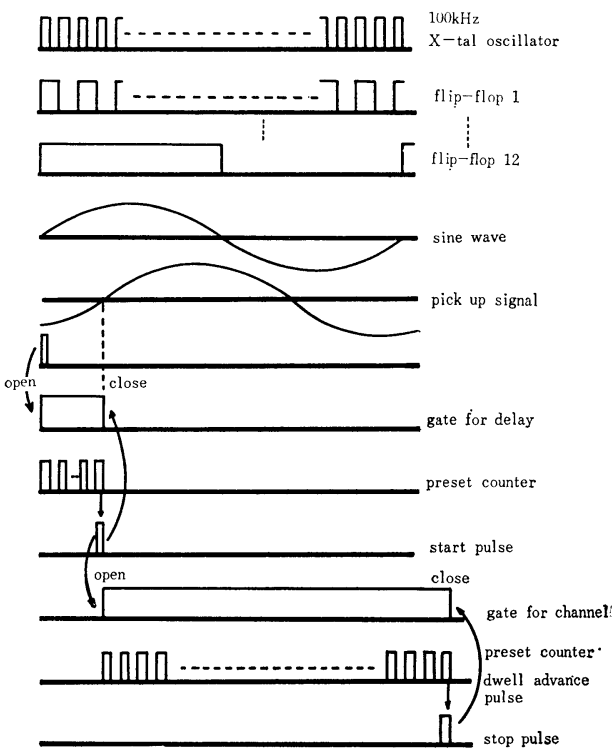

Fig. 3 A schematic time chart for pulses. in the MCA drive unit. 
Fig. 2 and Fig. 3, respectively. The crystal oscillator generates the clock pulse of 100 kHz. This pulse is divided into the clock pulses of $50,25,12.5$ and $6.25 \mathrm{kHz}$ by using the flip-flop. An exact sine wave is generated from the twelfth flip-flop involved in the parallel $\mathrm{T}$ circuit, of which $Q$ value is selected to be about 30 . When it needs to produce a symmetrical saw tooth wave, the output signal of the twelfth flip-flop is integrated, instead of using the parallel $\mathrm{T}$ circuit. On the other hand, one of the clock pulses of $50,25,12.5$ and $6.25 \mathrm{kHz}$ is selected in "Pulse duration" circuit (in Fig. 2), according to the channel number of the MCA. The selected clock pulse train is transmitted to the preset counter in the delay circuit. These pulses are counted digitally until the number of the clock pulses coincides with the preset number. Then, the start pulse is generated. The same sort of the operation is also performed in the preset counter in the channel number circuit, by which the channel number [is_designated. This preset counter generates the dwell advance pulse and the stop pulse.

Fig. 4 shows the result obtained with $\mathrm{Fe}$ absorber at room temperature. This result agrees well with those reported before ${ }^{3)}$.

The temperature coefficient of a sine wave

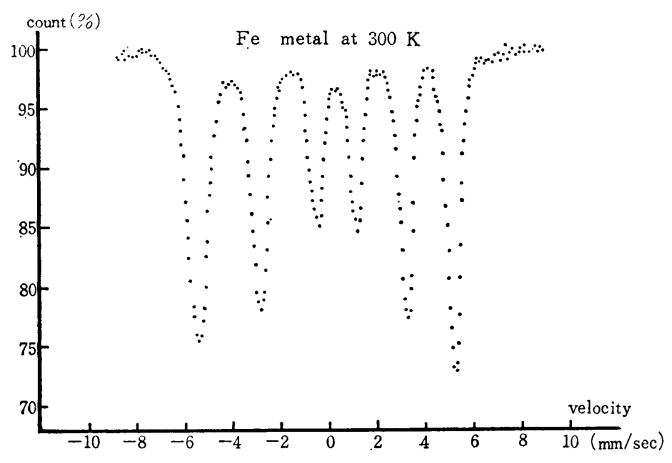

Fig. 4 Mössbauer spectrum obtained with ${ }^{57} \mathrm{Fe}\left({ }^{57} \mathrm{Co}\right.$ source embeded in a copper plate).

was negligibly small and the synchronization between the channels of the MCA and the velocity drive signal was performed. The details of the apparatus will be described elsewhere.

\section{References}

1) E. Krankeleit: Rev. Sci. Instr., 35, 194 (1964)

2) R.L. Cohen: Rev. Sci. Instr., 37, 260 (1966)

3) Goldanskii and Herber: Chemical Applications of Mössbauer Spectroscopy, Academic press, New York and London (1968) 